\title{
La gamificación como herramienta educativa: el estudiante de contabilidad en el rol del gerente, del contador y del auditor
}

\author{
Eloy González-Acosta ${ }^{1 *}$, Maylié Almeida-González ${ }^{1}$, Aleymis Torres-Chils ${ }^{2}$ y Yeny M. Traba-Montejo ${ }^{3}$ \\ (1) Universidad de Guayaquil, Facultad de Ciencias Administrativas, Carrera de Contaduría Pública Autorizada, Ciudadela \\ Universitaria, Guayaquil, Ecuador. (correo-e: eloygez@gmail; maylie.alm@gmail.com) \\ (2) Universidad de Guayaquil, Facultad de Ciencias Médicas, Carrera de Enfermería, Ciudadela Universitaria, Guayaquil, \\ Ecuador. (correo-e: atorreschils@gmail.com) \\ (3) Asociación de Profesores de Inglés del Ecuador. Pio Montufar 1134 y Capitán Nájera, Guayaquil, Ecuador. \\ (correo-e: trabayeny@gmail.com)
}

* Autor a quien debe ser dirigida la correspondencia

Recibido Ene. 27, 2020; Aceptado Mar. 30, 2020; Versión final Abr. 23, 2020; Publicado Oct. 2020

\begin{abstract}
Resumen
Esta investigación propone un aprendizaje por gamificación en un juego de rol con estudiantes de contabilidad que enfrentan dentro del aula una situación empresarial. Se estudian los resultados donde grupos de tres estudiantes asumen el papel del gerente, el contador y el auditor interno; y toman decisiones vinculadas a la gestión y a la ética. Comparativamente se analizó otro grupo de estudiantes que recibieron contenidos por métodos tradicionales. Simultáneamente se aplicó un cuestionario tipo Likert para medir la motivación en todos los estudiantes. Mediante un cuasiexperimento contrastado por una ANOVA factorial, se buscaron diferencias en las calificaciones por la incidencia del factor metodológico y el motivacional. Los resultados mostraron diferencias significativas para ambos factores. Se concluye que existe una relación entre las calificaciones de los estudiantes de contabilidad y el desarrollo de contenidos propios de la carrera a través del juego de rol propuesto.
\end{abstract}

Palabras clave: gamificación; juego de rol; competencia; habilidades docentes

\section{Gamification as an educational tool: the accounting student in the role of the manager, the accountant, and the auditor}

\begin{abstract}
This research proposes learning by gamification in a role-playing game with accounting students who face a business situation in the classroom. Groups of three students assume the role of the manager, the accountant, and the internal auditor and make management and ethical decisions. Comparatively, another group of students who received content by traditional methods was analyzed. A Likert-type questionnaire was applied to measure motivation in all students. A factorial ANOVA quasi-contrasted experiment was used to assess score differences in the incidence of the methodological and the motivational factors. The results showed significant differences for both factors. It is concluded there exist a correlation between the grades of students and the development of their own accounting career content by the proposed role-playing gamification.
\end{abstract}

Keywords: gamification; role playing; competence; teaching skills 


\section{INTRODUCCIÓN}

El cambio cualitativo que persigue la educación superior en la búsqueda de una alta calidad académica se fundamenta en generar mejores competencias de cara al mercado laboral. Lo que significa formar en el estudiante conocimientos sólidos con habilidades y destrezas dentro de su campo profesional; pero sobre todo actitud y valores humanos (Alonso et al., 2019; Calabor et al., 2018; Mabusela y Adams, 2016; Ros y Conesa, 2013). Este proceso formativo permanente se realiza en las condiciones de nuevos y complejos escenarios, que imponen renovar y dinamizar los procesos en la búsqueda de nuevos métodos de enseñanza y aprendizaje (Pourghaznein et al., 2015). Como resultado de estas estrategias educativas, los individuos están en condiciones de desarrollar una formación continua a lo largo de la vida y mantener un alto nivel de empleabilidad (Roig et al., 2011).

Aparece un rompimiento en el sistema tradicional donde el profesor es el actor principal y único en el proceso del aprendizaje y es más evidente la importancia de la activa participación de los estudiantes (Rodríguez et al., 2012). De forma que quedan obsoletos los métodos de enseñanza tradicionales con una base memorística, donde el estudiante solamente escucha las explicaciones y los ejemplos con una participación regulada y poca motivación (Topîrceanu, 2017). Molano et al., (2019) y Calabor et al., (2018) concuerdan en que mientras más activo sea el estudiante en su propio aprendizaje mejores resultados pueden esperarse del proceso formativo, en el que influyen además las exigencias de los constantes cambios socioeconómicos, culturales y sobre todo tecnológicos (Rodríguez et al., 2012). Específicamente la tecnología ha generado plataformas virtuales de aprendizaje, como Moodle o Chamilo, que transforman la docencia con nuevos recursos didácticos donde se pueden vincular los conceptos con la realidad (Calabor et al., 2018). Las tecnologías de la información y el conocimiento han permitido el surgimiento del e-learning como un método de aprendizaje basado en la web, que posibilita a todos aprender en cualquier momento y en cualquier lugar, sobre una base de autoaprendizaje (Pourghaznein et al., 2015).

A pesar de que hace varios años la investigación educativa ha profundizado en estos nuevos métodos de aprendizaje muchas universidades se mantienen impartiendo contenidos por sistemas tradicionales y la aplicación de formas alternativas es todavía limitada (Mabusela y Adams, 2016). Si bien es cierto que algunos docentes pueden ser capaces de impartir conferencias altamente motivacionales, este estilo puede ser poco fructífero en la construcción de asociaciones de los contenidos de alto nivel y en facilitar a los estudiantes los conocimientos profesionales que necesita (Randi y Carvalho, 2013). Según Duff (2004) el aprendizaje es influenciado por la percepción que asuma el estudiante de los requisitos de la tarea, que a su vez están marcados por las experiencias educativas anteriores y el contexto actual del aprendizaje. Es primordial que los docentes universitarios estén dispuestos a crear didácticas innovadoras diferentes a las tradicionales, capaces de atraer a un estudiante que maneja información y tecnología, busca participación y protagonismo y exige inmediatez. Esto puede implicar mayor esfuerzo y preparación del docente en generan metodologías más constructivistas donde tiene que escuchar, aprender y compartir la acción formativa (Gaete, 2011).

La gamificación dentro del aprendizaje consiste en la utilización de mecánicas de juego desarrolladas en entornos ajenos al juego, metodología que permite trabajar aspectos como la motivación, el esfuerzo y la cooperación dentro del ámbito escolar, estimulando a los alumnos, de tal manera que conduzca a una expansión de sus conocimientos (Prieto Andreu, 2020; Zainuddin et al., 2020). Estas metodologías brindan un enfoque eficaz para el aprendizaje centrado en el estudiante, generando las habilidades de construir y adquirir conocimientos; y desarrollar la actitud en un mundo de juego creado específicamente para fines educativos (Eugenio y Ocampo, 2019). Las propuestas docentes basadas en gamificación constituyen una excelente herramienta pedagógica, con objetivos formativos de alcanzar actitudes y aptitudes en los estudiantes similares a los que se esperan de los modelos basados en competencia; pero en su caso adquiridos dentro de una didáctica de retos y representaciones. De esta forma pueden incorporarse a los modelos educativos como elemento auxiliar que favorece la formación de competencias o resultados integrales, trabajado desde la diversión y la participación del estudiante (Hernández-Horta et al., 2018).

Podemos, por tanto, establecer la gamificación como la estrategia de utilizar el juego con otros fines que el puro entretenimiento, enfocada en alcanzar objetivos con la aplicación de herramientas lúdicas. En el caso educativo puede definirse una gamificación del aprendizaje, donde mediante juegos se busca incidir en la formación de los estudiantes, lo que algunos autores han tratado como juegos serios (Calabor et al., 2018). Una de estas herramientas que puede utilizarse en el aula dentro de la gamificación del aprendizaje es el juego de rol. Se trata de una actividad recreativa de representación de personajes, donde el estudiante puede teatralizar situaciones y que, en específico dentro de la educación superior, puede consistir en la simulación de situaciones propias de su futura profesión. Por tanto, consideramos que el desarrollo de un juego de rol en el aula con propósitos pedagógicos puede enmarcarse como una estrategia de gamificación orientada hacia el aprendizaje. 


\section{OTROS ANTECEDENTES}

Desde un punto de vista pedagógico, las estrategias de gamificación plantean un entorno donde tanto el contenido como la metodología influyen en la mejor adquisición de conocimientos y habilidades prácticas y en la resolución de desafíos y problemas. Desarrollado a partir de un aprendizaje que se enfoca al logro, donde se aprende de forma divertida e interesante y el estudiante se estimula al crear, comprender y retener información nueva, hasta el punto es capaz de aprender por sí mismo y de forma colaborativa (Alonso et al., 2019; Hernández-Horta et al., 2018). Según Svensson y Regnell (2017) y Ormazábal et al., (2019) el aprendizaje mediante gamificación permite a los estudiantes obtener conocimientos sobre hechos, ejemplos concretos de conceptos abstractos y capacidad de análisis en un ambiente de experiencia procesal y de toma de decisiones; así como la aplicación del contenido del curso en situaciones de la vida real.

Según Safapour et al., (2019) establecer un ambiente competitivo y de puntuaciones incide en que los estudiantes se esfuercen y participen activamente en el proceso de aprendizaje, movidos para una motivación intrínseca que genera mayor satisfacción e interés en las actividades. Aries et al., (2020) determina una relación entre el juego y el comportamiento, las emociones y el desarrollo cognitivo del estudiante. Como se dijo anteriormente, estas estrategias han estado influenciadas por los avances tecnológicos que relacionan el juego con dispositivos y plataformas con los cuales los estudiantes están cada vez más familiarizados (Sánchez et al., 2020; Hernández et al., 2014). Sin embargo, herramientas más utilizadas como el juego de rol dentro del aula, donde los estudiantes escenifican situaciones en las que asumen determinados personajes son también un claro ejemplo de gamificación.

Si bien en la teoría de Piaget (Piaget y Fernández, 1983) encontramos los conceptos psicológicos del proceso cognitivo, mediante el cual el individuo genera la capacidad para adaptarse e interiorizar la realidad que le rodeo, las teorías de David Kolb sostienen que el conocimiento se crea mediante la transformación de la experiencia, en la que el individuo aprende haciendo en un proceso de aprendizaje experiencial (Mabusela y Adams, 2016). Kolb establece cuatro estilos de aprendizaje que personalizan el enfoque del docente y su relación con el estudiante: el individuo activo, el reflexivo, el teórico y el pragmático (Jiménez Álvarez et al., 2019). Estos estilos se describen como un ciclo de cuatro etapas, que comienza cuando el individuo emprende una determinada tarea, mira atrás hacia su experiencia y reflexiona sobre ella; y luego concreta conceptos y generaliza para mejorarla (Fominykh et al., 2017). Se puede considerar como un enfoque al procesamiento de la información cognitiva, que describe las capacidades del alumno para operar las cuatro etapas u operar preferentemente en una de ellas (Duff, 2004). Según Poore et al., (2014) el aprendizaje es un ciclo continuo y recurrente; cada vez que el estudiante completa el ciclo de Kolb, el aprendizaje alcanza un nivel mayor de complejidad. Durante el juego de rol, como técnica de gamificación, el estudiante asume posiciones iniciales desde la experiencia anterior, reflexiona sobre la situación representada, participa de forma activa en un ambiente de experimentación; y crea individualmente conceptos y conocimientos propios enriqueciendo su experiencia.

Puede determinarse de esta manera el Juego de rol como una técnica dentro del enfoque experiencial de Kolb y como una excelente estrategia metodológica (Mabusela y Adams, 2016). Una herramienta que permitiría afrontar nuevas y complejas demandas formativas, orientada al desarrollo de competencias y a promover la capacidad de aprender a aprender, al involucrar al individuo en una interacción directa con aquello que estudia (Ferrero et al., 2018; Ros y Conesa, 2013). Estos tipos de juegos que simulan situaciones reales se han utilizado para recrear desde conflictos bélicos hasta el manejo de aeronaves, facilitando el planteamiento de estrategias y el desarrollo de las capacidades humanas más complejas (Alonso et al., 2019). En un juego de rol los participantes asumen un papel en una situación específica o escenario, de su propia parte o de otra persona, dentro de un ambiente seguro en el que puedan actuar, experimentar, aprender y enseñar (Connolly et al., 2009). Con la simulación de situaciones reales se genera un espacio de aprendizaje en el que cada jugador se comporta y prueba comportamientos como parte del entorno social y sus preferencias cognitivas, pero a la vez estudia el comportamiento de otros miembros y del equipo en su conjunto y de esta manera reconoce y evalúa condiciones que de otro modo no le eran evidentes (Islam y Islam, 2012; Kayes et al., 2005).

Por otro lado, el trabajo en equipo genera correcciones en el individuo al comparar sus conocimientos y comportamientos con el resto del grupo, lo cual mejora los resultados (Pérez, Cruz y Santana, 2007). Dado que los estudiantes no temen a las consecuencias personales de su comportamiento, son menos cautelosos y están menos inhibidos a la complejidad de la situación, este estado emocional de seguridad podría animarlos a aprender (Wesselow y Stoll-Kleemann, 2018; Connolly et al., 2009). Para esto es importante que el docente se sienta cómodo con el juego, gestione apropiadamente los tiempos, los equipos y haga una asignación correcta de papeles (Baruch, 2006). Adicionalmente Gordon y Thomas (2018) defienden la necesidad que el juego se realice con una planificación cuidadosa y la preparación para los jugadores y de los observadores y concluya cada ronda con una discusión posterior. 
En el campo de estudio de las ciencias administrativas, los juegos pueden definirse como un medio de formación con propósito educativo a través de una abstracción de un entorno empresarial, donde cada participante del juego debe asumir decisiones relacionadas con la gestión de la empresa o de otras áreas específicas (Alonso et al., 2019). Según Ros y Conesa (2013) el estudiante que egresa de la universidad debe estar vinculado a la realidad empresarial; poseer las competencias específicas, generales y transferibles con el conocimiento práctico; tener el carácter de un conocedor con habilidades claras y experimentadas en su campo con criterios profesionales. Para esto, según Duff (2004), dentro de la enseñanza de la contabilidad es necesaria la adopción de estrategias de aprendizaje apropiadas que conduzcan a mejorar el rendimiento académico y aumentar la capacidad del estudiante para aprender a aprender. Según Gaete (2011) los juegos de rol pueden considerarse en la formación de administradores vinculados a la conducta ética, el aprendizaje en teorías contables y financieras, las matemáticas y las estadísticas, la economía y el derecho. En específico, dentro de la enseñanza contable pueden aplicarse técnicas en las que se simulen la empresa, en un escenario donde los alumnos asumen el rol de contables o empresarios, con un impacto en la adquisición de las competencias propias del perfil profesional (Ros y Conesa, 2013).

Nuestro trabajo plantea una metodología de gamificación mediante el desarrollo de un juego de roles, para estudiantes de contabilidad que reciben la materia de auditoría en la facultad de Ciencias Administrativas de la Universidad de Guayaquil. El juego consiste en una simulación donde el estudiante de contabilidad, en una situación empresarial recreada y concreta, asume el papel del gerente financiero, el contador, o el auditor de la empresa. Se generan dos situaciones para cada trio de estudiantes donde se enfrentan a dos escenarios comunes en una organización. Cada alumno tendrá que tomar decisiones dentro del ámbito profesional y ético en un ambiente moderado por el profesor.

Lo novedoso de nuestra investigación es presentar una gamificación por juego de rol para estudiantes de contabilidad, simulando un ambiente de empresa u organización, para generar experiencias profesionales que impacten en su formación. Con el uso de herramientas estadísticas, se busca relacionar el factor metodológico y motivacional con los resultados académicos. En comparación con este trabajo, Ormazábal et al., (2019) plantea un juego de rol en estudiantes de farmacia que recrean la relación y la comunicación con pacientes. A diferencia de los trabajos de Ros y Conesa, (2013) y Calabor et al., (2018) que investigaron sobre el uso de juegos en estudiantes de contabilidad, nuestra investigación busca estudiar situaciones simuladas relacionadas con aspectos éticos y de gestión empresarial que generen discusión, valoración, reflexión y experimentación en el estudiante; y establecer un paralelismo y un soporte teórico en el aprendizaje experiencial de David Kolb.

\section{MÉTODO}

Se realizó un cuasiexperimento para determinar la relación causal entre las variables estudiadas. El estudio desea conocer si la interacción entre el factor del método de aprendizaje aplicado a estudiantes y el factor de sus niveles de motivación están relacionados con los resultados académicos. Las muestras utilizadas para las mediciones de las variables corresponden a los resultados de 249 estudiantes, separados en 8 grupos de la Carrera de contaduría pública autorizada de séptimo y octavo nivel, que representan el $33 \%$ de los estudiantes que cursan los 8 niveles de la carrera durante del periodo 2019-2020 en su Ciclo I. Los 8 grupos reciben una materia de auditoría acorde con su nivel. Para la enseñanza de los contenidos de las materias se aplicó en 4 de ellos un método de aprendizaje mediante el desarrollo de un juego de rol (grupos experimentales), los otros 4 grupos se mantuvieron recibiendo contenidos por un método tradicional de clases magistrales y casos prácticos (grupo control).

La asignación de los grupos correspondió a la clase y aula a los que está incluido cada estudiante por matriculación y que no se ha modificado en la investigación, o sea se han mantenido intactos sin selección o asignación aleatoria. Se desarrolló en los grupos experimentales un aprendizaje mediante la didáctica de un juego de rol donde los estudiantes asumieron uno de tres papeles: gerente financiero, contador o auditor interno de una pequeña y mediana empresas (pyme). Se trata de ocupaciones dentro del mundo de los negocios que puede ocupar un contador y son por lo tanto elementos importantes dentro de su perfil de egreso. Se dividió cada grupo en equipos de 3 estudiantes donde por sorteo se asignará uno de los tres papeles. De esta manera, se desarrolló una representación teatralizada de una temática desarrollada por los tres estudiantes y observada por el resto del grupo. Se diseñaron dos juegos que se aplicaron en dos rondas: un primer juego orientado a una situación de fraude y el segundo a una situación de crisis financiera en la organización. La forma en que se diseña el juego aparece detallada en la Tabla 1. Las rondas se desarrollaron en dos semanas del período académico, utilizando 4 horas semanales. El estudiante se expuso a situaciones simuladas de ambientes empresariales de oficina donde tuvo además que realizar investigaciones y valoraciones propias sobre las definiciones y contenidos de la materia de auditoría, pero además sobre contabilidad, emprendimientos, administración de empresas u otros relacionados con su carrera. 
Tabla 1: Diseño del Juego de rol. Elaborado a partir de Fominykh et al., (2017) y Wills, (2011)

\begin{tabular}{|c|c|c|c|}
\hline Actividades de aprendizaje & Integrantes & Recursos & Evaluación \\
\hline $\begin{array}{l}\text { Paso 1: Formación de equipos de } 3 \\
\text { estudiantes. } \\
\text { Paso 2: Se asigna el papel a cada } \\
\text { estudiante. } \\
\text { Paso 3. Investigación autónoma en } \\
\text { relación con el tema. } \\
\text { Paso 4: Representación en el aula. }\end{array}$ & $\begin{array}{l}\text { Estudiantes: } \\
\text { 1. Estudiante en papel de } \\
\text { gerente financiero. } \\
\text { 2. Estudiante en el papel de } \\
\text { contador. } \\
\text { 3. Estudiante en el papel de } \\
\text { auditor interno. } \\
\text { Docente: } \\
\text { 1.docente como moderador }\end{array}$ & $\begin{array}{l}\text { El espacio del } \\
\text { aula simulando } \\
\text { la oficina. }\end{array}$ & $\begin{array}{l}\text { La evaluación se } \\
\text { realizará en base a } \\
10 \text { puntos sobre los } \\
\text { contenidos } \\
\text { dispuesto para la } \\
\text { materia en el } \\
\text { semestre. }\end{array}$ \\
\hline
\end{tabular}

No se trata de un juego competitivo donde se establecen puntajes, se entregan insignias o estrellas por logros. El objetivo es que el alumno sea capaz de construir los conceptos desde la experiencia de la situación y se forme un criterio valorativo personal, jugando a actuar dentro de su personaje u observado otra representación. Después de cada recreación, el docente moderador generó además una discusión de resultados con los estudiantes que participaron con el resto del aula. El profesor orientó el análisis a temas como la ética, la gestión y el compromiso profesional que permitan relacionar y concretar conceptos. La comprensión de estos temas se evaluó posteriormente en un examen. En la Tabla 2 aparecen descritos el tema y el desarrollo del juego en sus dos rondas.

Tabla 2: Desarrollo del Juego de rol. Elaborado a partir de Fominykh et al., (2017) y Wills, (2011)

\begin{tabular}{|c|c|c|}
\hline Descripción del juego & Escenario & Objetivos de aprendizaje \\
\hline $\begin{array}{l}\text { Ronda 1. La actitud } \\
\text { ante el fraude. ¿Eres } \\
\text { de los nuestros? }\end{array}$ & $\begin{array}{l}\text { En una pyme con resultados anuales } \\
\text { favorables el gerente financiero propone al } \\
\text { contador y al auditor interno la alteración } \\
\text { de cifras en los estados financieros para } \\
\text { disminuir el pago de impuestos en favor de } \\
\text { los dueños. El gerente desafía el } \\
\text { compromiso con la organización con el } \\
\text { contador y el auditor interno ¿Eres de los } \\
\text { nuestros? El contador y el auditor tienen } \\
\text { que asumir una posición. }\end{array}$ & $\begin{array}{l}\text { 1. Desarrollar en los estudiantes valores } \\
\text { éticos y colocarlos en la situación de } \\
\text { decidir ante la propuesta del fraude. } \\
\text { 2. Relacionar los valores éticos con los } \\
\text { principios del código de ética del } \\
\text { contador y el marco de información } \\
\text { aplicable. } \\
\text { 3. Desarrollar habilidades de detección de } \\
\text { riesgos de auditoría y presentación de la } \\
\text { información financiera. }\end{array}$ \\
\hline $\begin{array}{l}\text { Ronda 2. La actitud } \\
\text { ante la crisis. ¿Cuál } \\
\text { es la solución? }\end{array}$ & $\begin{array}{l}\text { En una pyme con resultados anuales } \\
\text { desfavorables el Gerente Financiero } \\
\text { evalúa los resultados anuales y busca } \\
\text { respuesta en el contador y auditor. El } \\
\text { gerente desafía a la búsqueda de } \\
\text { alternativas para mejorar los resultados } \\
\text { ¿Cuál es la solución? El contador y el } \\
\text { auditor tienen que involucrarse en la } \\
\text { gestión desde su perspectiva. }\end{array}$ & $\begin{array}{l}\text { 1. Desarrollar en los estudiantes valores y } \\
\text { pensamiento crítico de compromiso y } \\
\text { proactividad. } \\
\text { 2. Desarrollar capacidades de análisis, } \\
\text { comparación y proyección de } \\
\text { resultados. } \\
\text { 3. Desarrollar habilidades relacionadas con } \\
\text { la administración y el manejo de } \\
\text { procesos. }\end{array}$ \\
\hline
\end{tabular}

La gestión formativa de los estudiantes en un parcial académico se imparte y evalúa en dos componentes: docencia y práctica, cada uno con un valor de 10 puntos. Para los grupos experimentales el componente de docencia se llevó a cabo con la presentación de clases magistrales, mientras los contenidos de la parte práctica se trataron con el desarrollo del juego de rol. En los grupos control el componente de docencia, al igual que el grupo experimental, se dictaron clases magistrales, sin embargo, el componente práctico se desarrolló mediante talleres y estudios de casos. Para ambos grupos, la evaluación del componente de docencia se realizó por la participación en las clases magistrales de los estudiantes mientras el componente práctico se evaluó mediante un examen de 10 preguntas con opción múltiple. Los grupos de control no recibieron contenidos vinculados con juegos de roles $u$ otras técnicas de aprendizaje. Las calificaciones de los estudiantes del examen de 10 preguntas correspondientes al componente práctico será nuestra variable para estudiar comparativamente las metodologías y su impacto en las calificaciones, sea un juego de rol o el uso de talleres y estudio de casos. Estas estrategias formativas y su evaluación se detallan en la Tabla 3.

Tabla 3: Componentes de la gestión formativa y su evaluación

\begin{tabular}{|l|l|l|l|l|}
\hline Grupo & $\begin{array}{l}\text { Actividades } \\
\text { componente docente }\end{array}$ & $\begin{array}{l}\text { Evaluación componente } \\
\text { docente }\end{array}$ & $\begin{array}{l}\text { Actividades componente } \\
\text { práctico }\end{array}$ & $\begin{array}{l}\text { Evaluación componente } \\
\text { práctico }\end{array}$ \\
\hline $\begin{array}{l}\text { Grupo } \\
\text { experimental }\end{array}$ & Clases magistrales & $\begin{array}{l}\text { Participación en clases } \\
(10 \text { puntos })\end{array}$ & $\begin{array}{l}\text { Estrategia de Gamificación } \\
\text { (Juego de rol) }\end{array}$ & $\begin{array}{l}\text { Examen de 10 preguntas } \\
\text { de opción múltiple. }\end{array}$ \\
\hline Grupo Control & Clases magistrales & $\begin{array}{l}\text { Participación en clases } \\
(10 \text { puntos) }\end{array}$ & Talleres y estudio de casos & $\begin{array}{l}\text { Examen de 10 preguntas } \\
\text { de opción múltiple. }\end{array}$ \\
\hline
\end{tabular}


Se determinó el nivel de motivación de los estudiantes al elaborar y aplicar un cuestionario tipo escala de Likert de 12 preguntas con opciones de respuesta de 1 a 5 (nunca, casi nunca, a veces, casi siempre y siempre). La suma de los resultados del cuestionario se agrupa en cinco categorías por separado (muy desfavorable, desfavorable, indiferente, favorable y muy favorable); que establecen el nivel de motivación del estudiante. El cuestionario que se describe en la Tabla 4 muestra el instrumento elaborado y ajustado a partir de las sugerencias y cambios propuestos por seis docentes consultados con amplia experiencia en investigación relacionada a las ciencias de la educación, los cuales revisaron la estructura y contenido del cuestionario; así como la pertinencia de las preguntas. La motivación es un constructo hipotético, que no pueden ser observados directamente, pero que han revelado tener un importante poder explicativo y predictivo en las conductas humanas, y, por supuesto, en las adoptadas por los estudiantes (Delgado y Castrillo, 2015). Para evaluar la fiabilidad de los resultados obtenidos en los cuestionarios aplicados se calcula el Alfa de Cronbach.

Tabla 4: Cuestionario tipo Likert para la medición de la Motivación

\begin{tabular}{|l|l|}
\hline \multicolumn{2}{|l|}{ Motivación hacia el aprendizaje } \\
\hline Código & Pregunta \\
\hline M01 & Decidí estudiar esta carrera para trabajar en algo que me gusta \\
\hline M02 & Estudio para aumentar mis posibilidades en el mercado de trabajo \\
\hline M03 & Estudio para aprender y avanzar en mis conocimientos \\
\hline M04 & Me motiva comprobar que soy capaz de superarme a mí mismo \\
\hline M05 & Prefiero llevar una vida ordenada, organizada \\
\hline M06 & Estudio para educarme, para madurar como persona \\
\hline M07 & Estudio porque así puedo vivir con más independencia \\
\hline M08 & Estudio por vocación, porque siempre quise estudiar esta carrera \\
\hline M09 & Estudio porque me hace sentirme bien conmigo mismo/a \\
\hline M10 & Estudio para conocer gente nueva y entablar amistades con chicos como yo \\
\hline M11 & Mis profesores y su metodología me motivan para seguir estudiando \\
\hline M12 & Estudio en la universidad porque me divierto también mucho \\
\hline
\end{tabular}

Nuestra pregunta de investigación consistió en: ¿existen diferencias significativas en las calificaciones de los estudiantes dependiendo del método de aprendizaje que se utilice y de su nivel de motivación? Esta pregunta se ha sistematizado con las siguientes interrogantes: 1) ¿Existen diferencias significativas en las calificaciones de los estudiantes según la aplicación de método de aprendizaje tradicional o un método experiencial de juego de rol?, 2) ¿Existen diferencias significativas en las calificaciones de los estudiantes según su nivel de motivación en los niveles de alto, medio y bajo?, 3) ¿Existen diferencias significativas en las calificaciones de los estudiantes según el método de aprendizaje que se utilice en interacción con el nivel de motivación del estudiante? Se procedió a realizar la prueba de estadística de Análisis de Varianza (ANOVA factorial) que permitió establecer diferencias por separado y en interacción de dos factores con una variable dependiente (Wang y DeVogel, 2019). Se buscó específicamente diferencias entre el tipo de método de aprendizaje aplicado (Variable independiente) y el nivel de motivación de los estudiantes (variable independiente) con sus resultados académicos (variable dependiente).

El análisis estadístico de los datos en general se realizó con el Software estadístico SPSS V23, utilizado para el análisis de fiabilidad el Alfa de Cronbach en el estudio y la agrupación de la escala de Likert. Se utilizó el software además para la comprobación de los supuestos propios de las pruebas paramétricas en cuanto normalidad de la distribución y homocedasticidad en relación con las varianzas de los grupos. Se utilizó también para la determinación de diferencias en el ANOVA Factorial analizada en el modelo lineal generalizado de tipo univariante y los análisis de diferencias entre grupos comparados mediante el Test de Bonferroni. Se estableció el análisis estadístico bifactorial teniendo en cuenta la imposibilidad de manejar la investigación como un experimento puro. Si bien nuestro estudio está enfocado al impacto del método de aprendizaje se considera que la motivación del estudiante también tendría un efecto en el diseño por lo cual se incorpora como factor a estudiar y se selecciona el estadístico factorial.

\section{RESULTADOS Y DISCUSIÓN}

A los resultados de la aplicación del cuestionario para obtener información sobre el nivel de motivación de los estudiantes se le aplicó el coeficiente del Alfa de cronbach, el análisis arrojó un resultado de 0,86 que muestra como buena la consistencia interna de los datos y por tanto su fiabilidad. Según la prueba KolmogorovSmirnov, que se detalla en la Tabla 5, se observa que los datos correspondientes a las notas obtenidas por los estudiantes se distribuyen normalmente para la variable método, en los dos grupos formados por los dos 
niveles separados en los estudiantes que recibieron el contenido por aprendizaje por juego de roles y los estudiantes que lo recibieron un método de aprendizaje tradicional (Estadístico .070 y .058; p>05). Al aplicar de igual manera esta prueba a los datos de la variable calificación, pero en relación con los grupos de la variable motivación, desglosada en los niveles alto, medio y bajo; se observa también una distribución normal para los tres grupos (Estadístico .088, .081 y .076; p>.05).

Tabla 5. Pruebas de normalidad de los datos. Se utiliza Kolmogorov-Smirnov analizando el tamaño de la muestra. Diferencia significativa al nivel de 0.05

\begin{tabular}{|l|l|l|l|l|}
\hline \multicolumn{2}{|l|}{ Método } & \multicolumn{4}{|l|}{ Kolmogorov-Smirnov } \\
\cline { 3 - 5 } & Estadístico & gl & Sig. \\
\hline \multirow{2}{*}{ Calificación } & Tradicional &, 070 & 129 &, 200 \\
\cline { 2 - 5 } & Juego de Roles &, 058 & 120 &, 200 \\
\hline Calificación & Bajo &, 088 & 50 &, 200 \\
\cline { 2 - 5 } & Medio &, 081 & 74 &, 200 \\
\cline { 2 - 5 } & Alto &, 076 & 125 &, 074 \\
\hline
\end{tabular}

La prueba de Levene para establecer el supuesto de homocedasticidad indica que existe igualdad de varianzas entre los grupos del diseño ( $F: 1.075 ; p>.05)$. Al comprobar la distribución normal en los grupos estudiados; así como la igualdad de las varianzas establecemos como verdaderos los supuestos para realizar una prueba estadística paramétrica que en este caso será la aplicación de una ANOVA factorial. Como se detalla en la Tabla 6 , de acuerdo con el ANOVA factorial se pueden identificar que hay diferencias en las calificaciones obtenidas de acuerdo con el método de aprendizaje que se utiliza ( $F: 207.860 p<.001$ ), de igual manera existen diferencias en las calificaciones obtenidas de acuerdo con el grado de motivación que presentan los estudiantes separado en bajo, medio y alto ( F: $9.914 p<.001)$. Sin embargo, el diseño aplicado nos muestra que la interacción entre el método y la motivación no causan diferencias en la variable dependiente.

Tabla 6: Prueba de efectos intersujetos del ANOVA Factorial. La diferencia de medias es significativa al nivel 0,05.

\begin{tabular}{|l|l|l|l|l|l|}
\hline Origen & Suma de cuadrados & gl & $F$ & Sig. & Potencia observada \\
\hline Modelo corregido & 62,213 & 5 & 53,629 &, 000 & 1,000 \\
\hline Intersección & 14654,549 & 1 & 63162,862 &, 000 & 1,000 \\
\hline Método & 48,226 & 1 & 207,860 &, 000 & 1,000 \\
\hline Motivación & 4,600 & 2 & 9,914 &, 000 &, 983 \\
\hline Método * Motivación &, 294 & 2 &, 634 &, 532 &, 155 \\
\hline Error & 56,379 & 243 & & & \\
\hline Total & 17093,390 & 249 & & & \\
\hline Total corregido & 118,592 & 248 & & & \\
\hline
\end{tabular}

Se aplica la prueba post-hoc de Bonferroni para establecer el nivel de significación de las diferencias comparando las medias de los grupos de motivación entre baja, media y alta. Como se aprecia en la Tabla 7 la prueba Bonferroni determina que es significativa las diferencias de las calificaciones para los estudiantes que presentaron una motivación alta en relación con los niveles medio y bajo $(p<.05)$. No se detectaron diferencias significativas en las notas de estudiantes de motivación media con estudiantes de motivación baja $(p>$. 05). Y finalmente, se observa que las calificaciones fueron superiores para los estudiantes de motivación alta con respecto a los estudiantes de motivación media y baja.

Tabla 7: Comparación múltiple por la prueba Bonferroni para la calificación entre los niveles de Motivación. La diferencia de medias es significativa al nivel 0,05.

\begin{tabular}{|l|l|l|l|l|l|l|}
\hline \multirow{2}{*}{ (I) Motivación } & (J) Motivación & Diferencia de medias (I-J) & Error estándar & Sig. & \multicolumn{3}{|l|}{ Intervalo de confianza al 95\% } \\
\cline { 3 - 7 } & & & & & Límite inferior & Límite superior \\
\hline \multirow{2}{*}{ Bajo } & Medio &, 0337 &, 08818 & 1,000 &,- 1789 &, 2462 \\
\cline { 2 - 7 } & Alto &,- 2164 &, 08060 &, 023 &,- 4107 &,- 0221 \\
\hline \multirow{2}{*}{ Medio } & Bajo &,- 0337 &, 08818 & 1,000 &,- 2462 &, 1789 \\
\cline { 2 - 7 } & Alto &,- 2501 &, 07065 &, 001 &,- 4204 &,- 0798 \\
\hline \multirow{2}{*}{ Alto } & Bajo &, 2164 &, 08060 &, 023 &, 0221 &, 4107 \\
\cline { 2 - 7 } & Medio &, 2501 &, 07065 &, 001 &, 0798 &, 4204 \\
\hline
\end{tabular}


Se comprobó que las calificaciones obtenidas con la aplicación del examen en base a 10 puntos y que evaluó los resultados de la formación práctica en los estudiantes, están relacionados con el tipo de metodología de aprendizaje aplicada. Esto significa que el uso de la gamificación por juego de rol tiene una incidencia en los conocimientos adquiridos en comparación con los resultados en los estudiantes que desarrollaron talleres 0 casos prácticos, que han sido las herramientas más comunes en la formación práctica de estudiantes de contabilidad. Se demostró además que los niveles de motivación que se establecieron en los estudiantes con la aplicación de un cuestionario tipo Likert, están también relacionados con los resultados del examen aplicado. No se puedo demostrar que la aplicación de las metodologías de aprendizajes y los diferentes niveles de motivación incidan de forma simultánea en las calificaciones aun cuando lo hagan por separado. A partir de relacionar el nivel de motivación con las diferencias en las medias de las calificaciones se comprobó que mientras más alta la motivación del estudiante en el aprendizaje mejores fueron sus calificaciones.

Como parte de la investigación de Calabor et al. (2018) sobre el impacto de juegos serios en la adquisición de competencias, se aplicaron cuestionarios en estudiantes de contabilidad que demostraron un aumento en la percepción del alumno de su capacidad de análisis e interpretación de los costes empresariales. Por otro lado, Ros y Conesa, (2013) concluyen que la simulación mediante juegos de rol parece ser adecuada para alcanzar un aprendizaje efectivo y eficaz, según la valoración hecha por estudiantes en relación con estrategias diseñadas para materias de Contabilidad Financiera y Análisis de los Estados Contables en alumnos de Administración y Dirección de empresas.

Según Alonso et al. (2019) el uso de juegos formativos permite desarrollar destrezas, asociar y conectar información, mejorar la capacidad de análisis en la formación administrativa y gerencial de los estudiantes. En su investigación sobre gamificación en la educación superior, Hernández-Horta et al., (2018) proponen un juego diseñado para fungir como herramienta multidisciplinaria complementaria a la enseñanza, una propuesta de juego puede adaptarse a cualquier rama de la ciencia. Con los resultados de estas investigaciones nuestro trabajo encuentra puntos coincidentes con relación al impacto del uso de los juegos en estudiantes de ciencias administrativas, aunque en nuestro estudio utilizamos una metodología diferente.

\section{CONCLUSIONES}

El desarrollo del cuasiexperimento con la aplicación de pruebas estadísticas permitió obtener a las conclusiones siguientes:

1) Existe una relación en las calificaciones de los estudiantes de contabilidad al desarrollar contenidos propios de la carrera a través de un juego de rol.

2) Se comprobó que el nivel de motivación de los alumnos está de igual manera relacionado con las calificaciones obtenidas, aunque no existe relación en la interacción de metodología y motivación simultáneamente con las notas de los estudiantes.

3) Los alumnos que presentaron una motivación alta obtuvieron una media de calificaciones superior a los que mantuvieron una motivación media-baja.

4) Con este trabajo se aporta evidencia empírica que presenta el juego de rol como método educativo acertado dentro del campo de la gamificación, capaz de generar el interés y motivación en el estudiante de contabilidad, que al participar activamente en el juego se vuelve formador de su propio aprendizaje.

5) Al encontrar significatividad en la relación entre el uso de un método de aprendizaje por gamificación con las calificaciones de los estudiantes, se comprueba la relevancia de situar al estudiante en un juego que simula un escenario empresarial, diferente al ambiente tradicional del aula y similar al de la vida profesional, con lo cual consideramos se aporta también a la literatura empírica del aprendizaje experiencial.

\section{REFERENCIAS}

Alonso, O., Palacios, S.M., y otros tres autores, Aprendizaje basado en juegos formativos: Caso Universidad en Colombia, doi:10.24320/redie.2019.21.e12.2024, Revista Electrónica de Investigación Educativa, 21(1), 1-20 (2019).

Aries, A., Vional V., y otros tres autores, Gamification in learning process and its impact on entrepreneurial intention, doi:10.5267/j.msl.2019.10.021, Management Science Letters, 10(4), 763-768 (2020).

Baruch, Y, Role-play Teaching: Acting in the Classroom, doi:10.1177/1350507606060980, Management Learning, 37(1), 43-61 (2006).

Calabor, M.S., Mora, A., y Moya, S., Adquisición de competencias a través de juegos serios en el área contable: Un análisis empírico, doi: 10.1016/j.rcsar.2016.11.001, Revista de Contabilidad - Spanish Accounting Review, 21(1), 38-47 (2018). 
Delgado, M. M., y Castrillo, L.Á., Efectividad del aprendizaje cooperativo en contabilidad: Una contrastación empírica. Doi :10.1016/j.rcsar.2014.05.003, Revista de Contabilidad, 18(2), 138-147 (2015).

Duff, A., The role of cognitive learning styles in accounting education: Developing learning competencies, doi: 10.1016/j.jaccedu.2003.09.004, Journal of Accounting Education, 22(1), $29-52$ (2004).

Eugenio, F.C., y Ocampo, A.J.T., Assessing Classcraft as an Effective Gamification App based on Behaviorism Learning Theory, doi: 10.1145/3316615.3316669, Proceedings of the 2019 8th International Conference on Software and Computer Applications - ICSCA '19, 325-329 (2019).

Ferrero, G., Bichai, F., y Rusca, M., Experiential Learning through Role-Playing: Enhancing Stakeholder Collaboration in Water Safety Plans, doi: 10.3390/w10020227, Water, 10(2), 227 (2018).

Fominykh, M., Leong, P., y Cartwright, B., Role-playing and Experiential Learning in a Professional Counseling Distance Course, Journal of Interactive Learning Research, 29(2), 169-188 (2017).

Gaete, R.A., University education: Role playing as a strategy for evaluating university learning, doi:10.5294/edu.2011.14.2.3, Educación y Educadores, 14(2), 289-307 (2011).

Gordon, S., y Thomas, I., The learning sticks: Reflections on a case study of role-playing for sustainability, doi:10.1080/13504622.2016.1190959, Environmental Education Research, 24(2), 172-190 (2018).

Hernández, M.R., Rodríguez, V.M., Parra, F.J., y Velázquez, P., Las tecnologías de la información y la comunicación (tics) en la enseñanza-aprendizaje de la química orgánica a través de imágenes, juegos y video, doi: 10.4067/S071850062014000100005, Formación universitaria, 7(1), 31-40 (2014).

Hernández-Horta, I.A., Monroy-Reza, A., y Jiménez-García, M., Aprendizaje mediante Juegos basados en Principios de Gamificación en Instituciones de Educación Superior, doi:10.4067/S0718-50062018000500031, Formación universitaria, 11(5), 31-40 (2018).

Islam, P., y Islam, T., Effectiveness of role play in enhancing the speaking skills of the learners in a large classroom: An investigation of tertiary level students, doi:10.3329/sje.v7i0.14475, Stamford Journal of English, 7, 218-233 (2012).

Jiménez, L.S., y otros cuatro autores, Estilos y estrategia de enseñanza-aprendizaje de estudiantes universitarios de la Ciencia del Suelo, doi:10.24320/redie.2019.21.e04.1935, Revista Electrónica de Investigación Educativa, 21 (1), 1 (2019).

Kayes, A.B., Kayes, D.C., y Kolb, D.A., Developing teams using the Kolb Team Learning Experience, doi:10.1177/1046878105279013, Simulation \& Gaming, 36(3), 355-363 (2005).

Mabusela, S., y Adams, J., Employing Role play in teaching and learning: A case of Higher Education, doi:10.20853/27-3263, South African Journal of Higher Education, 27(3), 489-500 (2016).

Molano, L.N., Mendoza, R.E., y Mendoza, H.H., Didáctica de la Competencia Gramatical mediada por Aprendizaje Activo en Estudiantes de una Licenciatura, doi: 10.4067/S0718-50062019000600167, Formación universitaria, 12(6), 167-182 (2019).

Ormazábal, V., Almuna, F., Hernández, L. y Zúñiga, F., Juego de roles como método de enseñanza de Farmacología para estudiantes de la carrera de enfermería, doi: 10.1016/j.edumed.2018.07.001, Educación Médica, 20(4), $206-212$ (2019).

Pérez, V.M., Cruz, N.M., y Santana, M.P.P., El uso de las nuevas tecnologías para favorecer el trabajo en equipo. La simulación estratégica como técnica de aprendizaje experimental, Conocimiento, innovación y emprendedores: camino al futuro, 1449-1465 (2007)

Piaget, J., y Fernández, F.J., Psicología y pedagogía, Ariel, Barcelona, España (1983).

Poore, J.A., Cullen, D.L., y Schaar, G.L., Simulation-Based Interprofessional Education Guided by Kolb's Experiential Learning Theory, doi: 10.1016/j.ecns.2014.01.004, Clinical Simulation in Nursing, 10(5), e241-e247 (2014).

Pourghaznein, T., Sabeghi, H., y Shariatinejad, K., Effects of e-learning, lectures, and role playing on nursing students' knowledge acquisition, retention and satisfaction, Medical Journal of The Islamic Republic of Iran, 29, 7 (2015).

Prieto, J.M., Una revisión sistemática sobre gamificación, motivación y aprendizaje en universitarios, doi:10.14201/teri.20625, Teoría de la Educación. Revista Interuniversitaria, 32(1), 73 (2020).

Randi, M.A.F., y Carvalho, H.F., Learning through role-playing games: An approach for active learning and teaching, doi:10.1590/S0100-55022013000100012, Revista Brasileira de Educação Médica, 37(1), 80-88 (2013).

Rodríguez, G., Ibarra, M.S., Gallego, B., Gómez, M.A., y Quesada, V., La voz del estudiante en la evaluación del aprendizaje: Un camino por recorrer en la universidad, doi:10.7203/relieve.18.2.1985, RELIEVE - Revista Electrónica de Investigación y Evaluación Educativa, 18(2), 2 (2012).

Roig, A.E., Salva, B.G., y González, I.G., Las nuevas culturas de aprendizaje y su incidencia en la educación superior, RMIE. Revista Mexicana de Investigación Educativa, 16(51) (2011).

Ros, M.I. y Conesa, M. del C., Adquisición de competencias a través de la simulación y juego de rol en el área contable, doi:10.5209/rev_ESMP.2013.v19.42049, Estudios sobre el Mensaje Periodístico, 19(0), 419-428 (2013).

Safapour, E., Kermanshachi, S. y Taneja, P., A Review of Nontraditional Teaching Methods: Flipped Classroom, Gamification, Case Study, Self-Learning, and Social Media, doi:10.3390/educsci9040273, Education Sciences, 9(4), 273 (2019). 
Sanchez, D.R., Langer, M., y Kaur, R., Gamification in the classroom: Examining the impact of gamified quizzes on student learning, doi:10.1016/j.compedu.2019.103666, Computers \& Education, 144, 103666 (2020).

Svensson, R.B., y Regnell, B., Is role playing in Requirements Engineering Education increasing learning outcome? doi:10.1007/s00766-016-0248-4, Requirements Engineering, 22(4), 475-489 (2017).

Topîrceanu, A., Gamified learning: A role-playing approach to increase student in-class motivation, doi: 10.1016/j.procs.2017.08.017, Procedia Computer Science, 112, 41-50 (2017).

Wang, T., y DeVogel, N., A revisit to two-way factorial ANOVA with mixed effects and interactions, doi: 10.1080/03610926.2019.1604961, Communications in Statistics - Theory and Methods, 1-18 (2019).

Wesselow, M., y Stoll-Kleemann, S., Role-playing games in natural resource management and research: Lessons learned from theory and practice, doi:10.1111/geoj.12248, The Geographical Journal, 184(3), 298-309 (2018).

Wills, S., The Power of Role-based e-Learning: Designing and Moderating Online Role Play, 1st edition, doi:10.4324/9780203842676, Routledge, New York, USA (2011).

Zainuddin, Z., Shujahat, M., Haruna, H., y Chu, S.K.W., The role of gamified e-quizzes on student learning and engagement: An interactive gamification solution for a formative assessment system, doi:10.1016/j.compedu.2019.103729, Computers \& Education, 145, 103729 (2020). 